\section{Impact of Methicillin-Resistant Staphylococcus aureus Infection on Morbidity and Costs in Healthcare Facilities}

T O THE EDIT OR-The research groups of Cosgrove et al. ${ }^{1}$ and Reed et al. ${ }^{2}$ should be applauded for their recently published work in this journal on defining the true costs of infection due to methicillin-resistant Staphylococcus aureus (MRSA). Their studies represent the state of the art on this topic and clearly show that MRSA infections result in increased morbidity (eg, longer hospitalization duration) and costs, compared with methicillin-susceptible $S$. aureus (MSSA) infections. In retrospect, much suffering and expense would have been avoided if the MRSA epidemic had been controlled at a much earlier stage, and in prospect, much suffering and expense can be avoided if the endemic is now seriously addressed. ${ }^{3}$ To further this cause, I suggest that the impact of these studies can be enhanced if 2 weaknesses they contain can be addressed.

Cosgrove et al. ${ }^{1}$ acknowledged in the discussion of their findings that their comparative methods, despite the careful and well-designed manner in which they were applied, were likely to overestimate the true effect of methicillin resistance on morbidity and costs. This is a problem of comparative studies, because it is not possible to completely avoid all selection biases, because of confounders hidden in the patient cohorts. Although this problem was not noted by Cosgrove and colleagues, it was addressed much earlier by Haley et al., ${ }^{4}$ who, because of hidden confounding, concluded that the costs of nosocomial infections are prone to be overestimated by methods that compare patients with nosocomial infection with patients without nosocomial infection. More-conservative estimates are obtained by means of noncomparative methods, such as the appropriateness evaluation protocol, in which the effect of infection with MRSA is ascertained for each patient individually. ${ }^{5}$ It would be very helpful if Cosgrove and colleagues included more-conservative estimates in their data set.

The study by Reed et al. ${ }^{2}$ also had a comparative design but largely avoided hidden selection biases, because the effect of MRSA infection on morbidity and costs was evaluated in a much more homogenous group of bacteremic patients (ie, those who were receiving long-term hemodialysis). Although hidden confounding can, by its nature, never be excluded completely, the homogeneity of the patient cohorts in this study was clearly evident, as shown in the patient and infection characteristics (tables 1 and 2). ${ }^{2}$ However, there is one important item that was presented but not accounted for. Patients with MRSA bacteremia had been hospitalized longer than patients with MSSA bacteremia before positive results of blood culture were obtained (mean duration, 2.5 vs 0.2 days; table 2). Although this difference was not statistically significant because of variation in the duration of hospitalization prior to positive results of blood culture, it may have had a highly significant impact on the outcome measures of the study (ie, mortality, duration of hospitalization, and costs). From their data in table 2, it can readily be estimated that, in the MSSA group, there was not one or only very few patients who did not receive a diagnosis of bacteremia within 1 day after admission. In sharp contrast, there must have been quite a few patients infected with MRSA (up to 25\% [13 patients!]) who received their diagnosis 2 or more days after admission.

Because Reed and colleagues calculated total inpatient costs (instead of those only incurred after the diagnosis, as was done by Cosgrove et al. ${ }^{1}$ ), the difference in the number of patients in whom infection was diagnosed within 1 day after admission significantly skewed their results toward overestimating the impact of methicillin resistance per se. The extent of the skew can easily be ascertained by adopting the methods of Cosgrove and colleagues or by recalculating the outcomes after excluding all patients with MRSA bacteremia who received their diagnosis after the last case of MSSA infection was recorded. If, after such censoring, a significant effect is still detected, this would certainly constitute one of the best pieces of evidence so far of the impact on morbidity and costs that the emergence of MRSA is having in healthcare facilities.

Henri A. Verbrugh, $M D, P h D$

From the Erasmus University Medical Center, Rotterdam, The Netherlands.

Address reprint requests to Henri A. Verbrugh, MD, PhD, Erasmus University Medical Center, Rotterdam, The Netherlands (h.a.verbrugh@ erasmusmc.nl).

Infect Control Hosp Epidemiol 2006; 27:994-995

(C) 2006 by The Society for Healthcare Epidemiology of America. All rights reserved. 0899-823X/2006/2709-0022\$15.00.

\section{REFERENCES}

1. Cosgrove SE, Qi Y, Kaye KS, et al. The impact of methicillin resistance in Staphylococcus aureus bacteremia on patient outcomes: mortality, length of stay, and hospital charges. Infect Control Hosp Epidemiol 2005; 26:166-174.

2. Reed SD, Friedman JY, Engemann JJ, et al. Costs and outcomes among hemodialysis-dependent patients with methicillin-resistant or methicillinsusceptible Staphylococcus aureus bacteremia. Infect Control Hosp Epidemiol 2005; 26:175-183.

3. Vos MC, Verbrugh HA. MRSA: we can overcome, but who will lead the battle? Infect Control Hosp Epidemiol 2005; 26:117-120.

4. Haley RW, Schaberg DR, Von Allmen SD, McGowan JE Jr. Estimating 
the extra charges and prolongation of hospitalization due to nosocomial infections: a comparison of methods. J Infect Dis 1980; 141:248-257.

5. Kim T, Oh PI, Simor AE. The economic impact of methicillin-resistant Staphylococcus aureus in Canadian hospitals. Infect Control Hosp Epidemiol 2001; 22:99-104.

\section{Polymyxins: A Word of Caution for Prudent Use of Valuable "Old Antibiotics"}

TO THE EDITOR-Infections due to gram-negative bacteria, such as Pseudomonas aeruginosa and Acinetobacter baumannii, that are resistant to most classes of the available antimicrobial agents are a rapidly growing, worldwide clinical problem that has a serious impact on mortality, morbidity, and healthcare-related costs. The lack of development of new antimicrobial agents to combat these infections, have made the medical community reevaluate the use of polymyxins that are old, almost abandoned antibiotics.

Both polymyxin $\mathrm{B}$ and polymyxin $\mathrm{E}$ (colistin) have recently been used for the treatment of patients with multidrugresistant gram-negative bacterial infections. The isolation of colistin from Bacillus colistinus was accomplished about 50 years ago. During the ensuing decades, colistin has been used in the treatment of several types of infections, including infectious diarrhea and urinary tract infection, as well as for bowel decontamination. Early clinical experience with polymyxins showed a high incidence of toxicity, mainly nephrotoxicity and neurotoxicity, including neuromuscular blockade. ${ }^{1}$ For example, in a large study of 288 patients, the incidences of nephrotoxicity and neurotoxicity after intramuscular administration of colistin were $20.2 \%$ and $7 \%$, respectively. ${ }^{1}$ Such data led to significant reduction in the systemic administration of polymyxins. During the past 2 decades, the use of polymyxins has mainly been restricted to topical ophthalmic and otic therapy, as well as treatment of pulmonary infections due to multidrug-resistant $P$. aeruginosa in patients with cystic fibrosis.

However, recent experience, including ours, with patients who have nosocomial infections due to multidrug-resistant gram-negative bacteria and who have been given intravenous polymyxins as a salvage therapy, suggests that polymyxins are valuable antimicrobial agents. ${ }^{2,3}$ Of note, the observed rates of nephrotoxicity among critically ill patients with infections caused by multidrug-resistant gram-negative bacteria who received intravenous colistin therapy were $8 \%$ and $14.3 \%$ in 2 recently published studies. Moreover, the comparison of the effectiveness and safety of intravenous colistin versus intravenous meropenem for the treatment of patients with ventilator-associated A. baumannii pneumonia revealed that the 2 therapeutic regimens yielded similar clinical responses; however, the rate of nephrotoxicity was considerably lower with colistin treatment. It is noteworthy that the incidence of aminoglycoside-induced nephrotoxicity has been reported to be $5 \%-25 \%$, which is not that different from the incidence observed in recent studies with intravenous colistin. ${ }^{4}$

We believe that polymyxins are life-saving antibiotics for patients with infections due to gram-negative bacteria resistant to all other available antimicrobial agents. However, the uncontrolled reintroduction of polymyxin therapy in several countries has led to the appearance of bacteria, mainly $P$. aeruginosa strains, that have developed mechanisms of resistance even to these agents. ${ }^{5}$ There is an urgent need for restriction of polymyxin use, to decrease the rate of emergence of really pandrug-resistant gram-negative bacteria-that is, bacteria with in vitro resistance to $\beta$-lactams, quinolones, aminoglycosides, tetracyclines, and polymyxins. Thus, polymyxins should be reserved for the treatment of patients with infections due to multidrug-resistant gram-negative bacteria when all other available antibiotic regimens have failed or patients with infections caused by microorganisms susceptible only to polymyxins.

Matthew E. Falagas, MD, MSc; Sofia K. Kasiakou, MD; Argyris Michalopoulos, MD

From the Alfa Institute of Biomedical Sciences (M.E.F., S.K.K., A.M.), the Department of Medicine (M.E.F.), and the Intensive Care Unit (A.M.), "Henry Dunant" Hospital, Athens, Greece; and the Department of Medicine, Tufts University School of Medicine, Boston, Massachusetts (M.E.F.).

Address reprint requests to Matthew E. Falagas, MD, MSc, Alfa Institute of Biomedical Sciences (AIBS), 9 Neapoleos Street, Marousi 151 23, Greece (m.falagas@aibs.gr).

Infect Control Hosp Epidemiol 2006; 27:995-995

(C) 2006 by The Society for Healthcare Epidemiology of America. All rights reserved. 0899-823X/2006/2709-0023\$15.00.

\section{REFERENCES}

1. Koch-Weser J, Sidel VW, Federman EB, Kanarek P, Finer DC, Eaton AE. Adverse effects of sodium colistimethate: manifestations and specific reaction rates during 317 courses of therapy. Ann Intern Med 1970; 72:857-868.

2. Falagas ME, Kasiakou SK. Colistin: the revival of polymyxins for the management of multidrug-resistant gram-negative bacterial infections. Clin Infect Dis 2005; 40:1333-1341.

3. Michalopoulos AS, Tsiodras S, Rellos K, Mentzelopoulos S, Falagas ME. Colistin treatment in patients with ICU-acquired infections caused by multiresistant gram-negative bacteria: the renaissance of an old antibiotic. Clin Microbiol Infect 2005; 11:115-121.

4. Smith CR, Lipsky JJ, Laskin OL, et al. Double-blind comparison of the nephrotoxicity and auditory toxicity of gentamicin and tobramycin. $N$ Engl J Med 1980; 302:1106-1109.

5. Falagas ME, Bliziotis IA, Kasiakou SK, Samonis G, Athanassopoulou P, Michalopoulos A. Outcome of infections due to pandrug-resistant (PDR) gram-negative bacteria. BMC Infect Dis 2005; 5:24. 